\title{
La cuisine : une technique, parfois un art, certainement pas une science de la nature
}

\section{Cooking: a technique, sometimes an art, never a science of nature}

\author{
Hervé This ${ }^{1,2}$ \\ ${ }^{1}$ Université Paris-Saclay, INRAE, AgroParisTech, UMR 0782 SayFood, 75005, Paris, France \\ ${ }^{2}$ Group of Molecular Gastronomy, Inrae-AgroParisTech International Centre for Molecular and Physical Gastronomy, \\ 16 rue Claude Bernard, F-75005, Paris, France
}

\begin{abstract}
RÉSUMÉ. Depuis les années 1980, la discipline scientifique nommée "gastronomie moléculaire et physique » est à l'origine de bouleversements des pratiques culinaires, par ses applications à la cuisine, mais si les personnes qui ont contribué à opérer les changements sont des scientifiques, elles sont sorties de leur rôle scientifique (la pratique de la gastronomie moléculaire) pour effectuer des travaux de transfert (l'application des sciences, c'est la technologie). L'ouverture technique qui en a résulté a conduit certains cuisiniers à mieux assumer le statut d'artistes culinaires, par opposition à ce qui est longtemps resté un artisanat ou un artisanat d'art. La première tendance artistique née des transferts techniques a été nommée " cuisine moléculaire "; elle a été suivie de diverses propositions et tentatives (cuisine abstraite, constructivisme culinaire, etc.), mais c'est surtout la cuisine de synthèse, surnommée " cuisine note à note ", qui se développe aujourd'hui dans le monde.
\end{abstract}

ABSTRACT. Since the 1980s, the scientific discipline called "molecular and physical gastronomy" has been responsible for upheavals in culinary practices, but the people who have contributed to the changes are scientists who have stepped out of their role to perform technological work. Technical openness due to these works has led some cooks to better assume the status of culinary artists, as opposed to what has long remained a craft or an art craft. The first trend born of technical transfers was called "molecular cuisine"; it was followed by various initiatives (abstract cuisine, culinary constructivism, etc.), but it is above all synthetic cooking, nicknamed "note-to-note cuisine", that is developing today throughout the world.

MOTS-CLÉS. gastronomie moléculaire et physique, cuisine moléculaire, cuisine de synthèse, cuisine note à note, transferts techniques, technologie culinaire.

KEYWORDS. molecular and physical gastronomy, molecular cuisine, synthetic cooking, note by note cooking, technical transfers, culinary technology.

Note liminaire : on pardonnera, $\mathrm{j}$ 'espère, l'aspect très personnel de cet article, en considérant que je sais mal discuter ce que je ne connais pas bien, alors que, au contraire, je peux témoigner de ce que je fais. D'ailleurs la fierté m'est étrangère, puisque je suis seulement intéressé par ce que je fais et ce que je ferai. On observera aussi que les travaux et résultats dont je fais état ici sont des dévoiements de mon activité scientifique : souvent des motivations politiques sous-tendent mes écarts à une recherche scientifique - la recherche en gastronomie moléculaire qui est mon intérêt principal (l'objectif n'est-il pas de lever un coin du grand voile de la nature ${ }^{1}$ ?). Et l'on pardonnera une insistance de ma part : je dirai en de nombreux endroits que la discipline scientifique qui a été nommée «gastronomie moléculaire » n'a en quelque sorte rien à voir avec la cuisine, ni à propos de la composante technique de cette dernière, ni a fortiori à propos de sa composante artistique. C'est seulement que la même personne prive son activité scientifique d'un temps précieux, pour avoir une action civique, au même titre que diverses conférences vers le grand public : certes, les scientifiques sont intéressés par l'exploration du monde, mais ne doivent-ils pas un peu de leur énergie à la société qui les finance? 


\section{L'art culinaire existe}

Cette courte mise en garde faite, il y a lieu de témoigner d'abord que, au début des années 2000, dans certains milieux intellectuels, les expressions «art culinaire » ou 《arts culinaires » étaient seulement des façons de parler imprécises, mais qui ne signifiaient en rien l'existence réelle de la cuisine en tant qu'art. D'ailleurs, la possibilité d'y mettre un pluriel était significative. En veut-on des preuves ? Le directeur de cabinet d'un ministre français, à la sortie d'une réunion avec ce ministre intéressé par l'enseignement des techniques et art culinaires m'a répondu que ces expressions étaient «quasi vides de sens », et que la cuisine n'avait «certainement » pas le même statut que la peinture, la musique, la littérature... Évidemment, une telle remarque montre seulement l'étroitesse d'un esprit particulier, mais je la cite, parce qu'elle faisait état d'un sentiment assez partagé.

Pourtant, à propos de ce que certains nomment des « aliments », et que je propose de nommer des plats, ou des mets pour distinguer «ce qui se mange » et «ce que l'on mange, parce qu'on l'a préparé », n'y a-t-il pas cette question essentielle du «bon»? Aucun d'entre nous ne souhaite évidemment manger quelque chose de «mauvais ». Or le bon, c'est le «beau à manger », tout comme il y a du beau à entendre, pour la musique, ou du beau à voir pour la peinture (on observera que je n'utilise évidemment pas de majuscule platonicienne à «bon» ou «beau»). Et il faut distinguer des formes différentes de cuisine : s'il y a, d'une part, une cuisine artisanale, toute de répétition, de «tradition», de «terroir», qui vise la satisfaction de besoins corporels, énergétiques, et qui s'apparente à la peinture en bâtiment, il y a, d'autre part, une cuisine artistique, pour laquelle la question n'est pas le contenu énergétique, ni la nature des nutriments, et qui s'apparente en tous points aux peintures d'un Matthias Grünewald, d'un Rembrandt, d'un Jérôme Bosch, aux musiques d'un Jean-Sébastien Bach, d'un Debussy... Oui, il y a de l'artisanat culinaire, d'un côté, et de l'art culinaire de l'autre ${ }^{2}$. Et il faut évidemment ajouter que, entre le noir et le blanc et il y a toute cette zone intermédiaire, avec notamment la question de l'artisanat d'art que nous ne discuterons pas ici.

L'ayant clairement compris, je ne cesse de l'expliquer au monde culinaire professionnel, dès le début des études (CAP, BEP...), afin que nos amis, surtout en formation initiale, puissent déterminer mieux l'activité qu'ils souhaitent avoir.

\section{Une initiative amicale}

Nous en arrivons au témoignage principal, qui est le suivant : en 1999, l'Académie des sciences m'avait invité à présenter la gastronomie moléculaire et physique (une discipline scientifique, nous y reviendrons), avant un dîner pour lequel on m'avait demandé de guider le traiteur ; mais, une semaine après cette invitation, le cuisinier français Pierre Gagnaire m'avait téléphoné, pour me rencontrer ; fut alors décidé un dîner «Science \& Art culinaires », où il mettrait « en art culinaire » certaines de mes «inventions $»^{3}$. Le succès de l'opération et, surtout, le plaisir que nous avions eu à travailler ensemble nous ont décidés de poursuivre nos travaux communs, avec des rendez-vous mensuels. Et c'est ainsi que, depuis 21 ans, je suis mis en position de donner à Pierre Gagnaire une invention chaque mois (Figure 1). Il n'est pas question d'argent, et le contrat amical entre nous est le suivant : chaque mois, sortant par amitié de mon activité de recherche en gastronomie moléculaire et physique, je cherche une application technique des connaissances que produit ma recherche scientifique, je la décris dans un article que je donne à Pierre Gagnaire pour qu'il le publie sur son site internet; en échange, si l'on peut dire, il travaille pour mettre mon invention en art culinaire, et 
il publie, à côté de mon article, entre une et quatre recettes qui font usage de ma proposition ; évidemment, il sert les recettes ainsi mises au point dans ses restaurants du monde.

L'objectif, avant tout amical, se double, pour ce qui me concerne, d'une volonté -parallèle à ma recherche scientifique en gastronomie moléculaire et physique- de rénover des techniques culinaires qui, dans les années 1980 encore, étaient très archaïques (on cuisinait à peu près comme au début du $20^{\mathrm{e}}$ siècle, ce qui doit évidemment conduire à nous interroger), mais, aussi, d'une volonté politique : je veux montrer que les sciences de la nature sont si fécondes qu'il n'est pas difficile d'en tirer une idée technique par mois ${ }^{4}$. Et je passe sur d'autres objectifs secondaires.

Quelques exemples pour fixer les idées. De l'évident, tout d'abord : un jour, ayant sous les yeux du sel, un mortier et un pilon, je me suis mis à broyer le sel, et j'ai produit ce que j'ai nommé du « sel glace». Jamais, auparavant, je n'avais vu un tel équivalent du sucre glace : c'est donc là une toute petite «invention», pour laquelle la science est quasi inutile, mais qui conduit à des perceptions intéressantes.
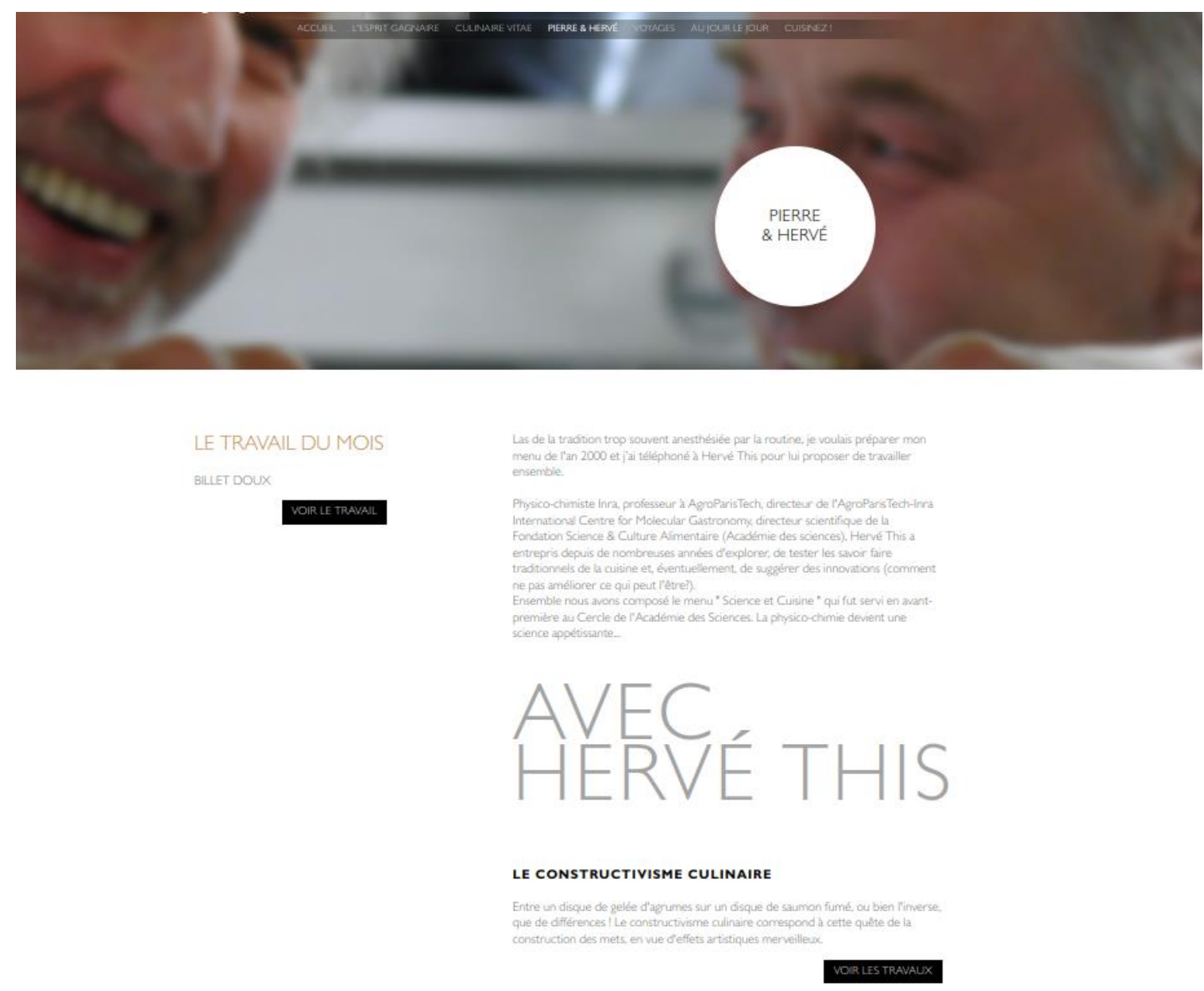

DICTONS, SAVOIR ET GOURMANDISE

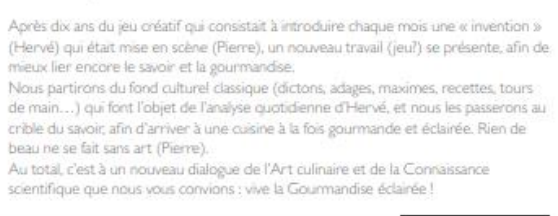

Figure 1. L'entrée du site de Pierre Gagnaire : la page renvoie vers (1) le travail du mois, (2) une liste de travaux dans la ligne " constructivisme culinaire ", (3) des textes qui explorent la " tradition culinaire " (" dictons, savoirs et gourmandise »), (4) des travaux de " cuisine note à note ». 
$\mathrm{Au}$ contraire, la gastronomie moléculaire et physique (une science de la nature, pas une technique) est à l'origine de ce que j'avais initialement nommé des «œufs parfaits » (Figure 2), aujourd'hui servis dans des restaurants du monde entier ${ }^{5}$. Initialement, il y avait une étude de la coagulation des œufs : en 1995, j'ai établi que cette coagulation résulte de l'établissement de ponts disulfures (en «décuisant» des œufs cuits) ${ }^{6}$. Toutefois ce premier résultat n'expliquait pas le fait (testé), qui est signalé par les cuisiniers : les œufs durs cuits plus que les dix minutes préconisées classiquement deviennent finalement «caoutchouteux », durs. L'analyse de la question a montré que la consistance des œufs dépend du nombre de protéines impliquées dans la formation du réseau solide qui fait le gel qu'est un œuf cuit; si l'on cuit, même longtemps, un œuf à la température de 65 ${ }^{\circ} \mathrm{C}$, alors très peu de protéines coagulent, et l'on obtient ce qui est donc un « œuf parfait », nom initial que j'ai ensuite proposé de changer pour « œuf à $65^{\circ} \mathrm{C}$ », puisque le parfait des uns n'est pas celui des autres, en supposant que la perfection puisse exister.

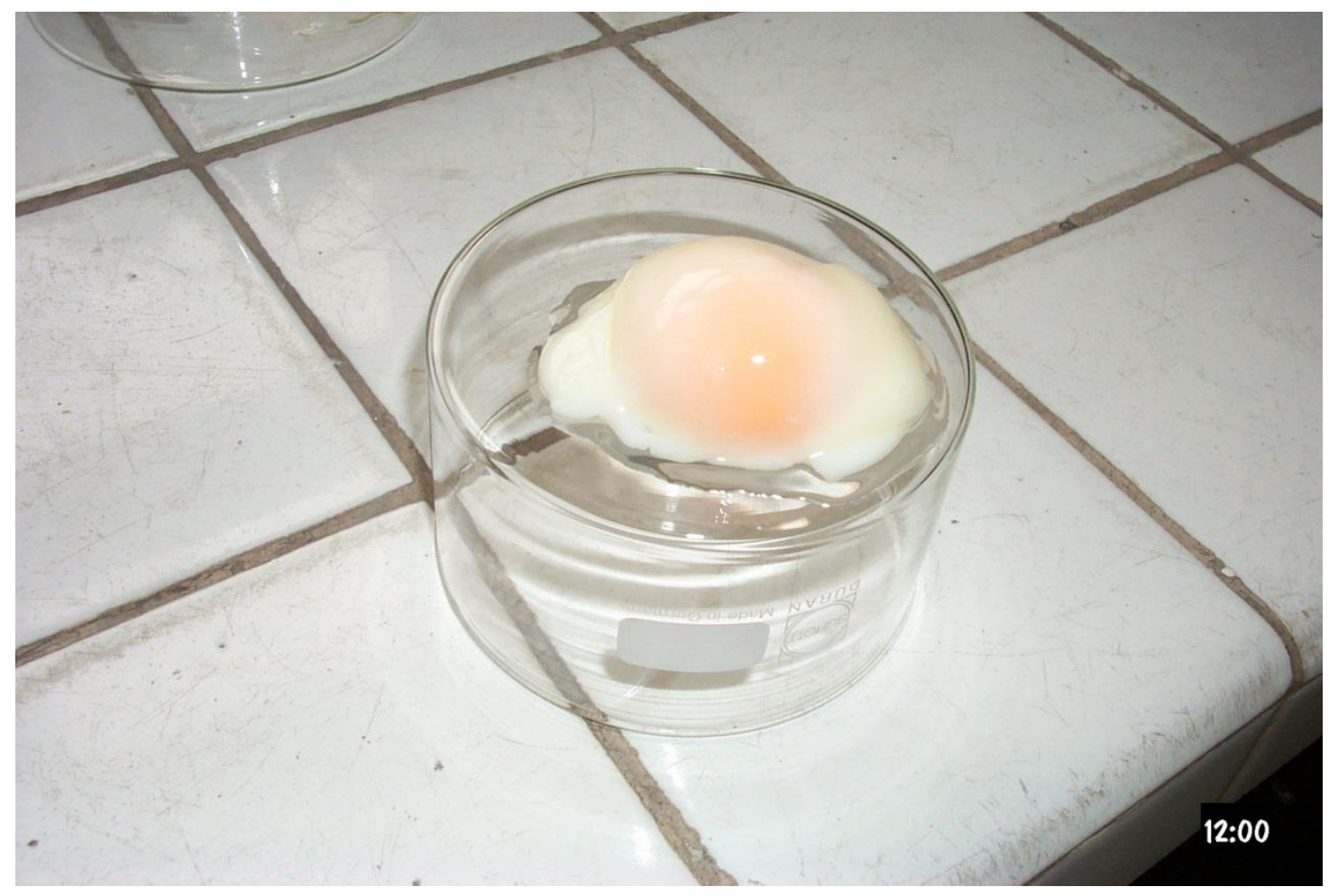

Figure 2. Un œuf à $65^{\circ} \mathrm{C}$ : à cette température, une seule sorte de protéines du blanc d'œuf coagule, ce qui forme un gel très délicat, opaque, et le jaune d'œu reste liquide, puisque la protéine qui coagule à 61,8 ${ }^{\circ} \mathrm{C}$ est en quantité insuffisante pour atteindre le seuil de percolation.

Il y a donc des transferts technologiques très variés, tant dans le degré d'innovation (un paramètre quantitatif s'impose, pour le mesurer) que dans l'importance des sciences de la nature pour l'introduction des innovations. Et il est très étonnant, pour un chimiste naïf, de voir que ce ne sont pas les innovations les plus «difficiles » qui sont les plus utiles, comme le montre l'exemple suivant, où fut résolu un problème technique posé par Pierre Gagnaire : ce dernier déplorait que le sel de Maldon (des cristaux sous formes de plaquettes, au croustillant apprécié) perde sa consistance entre le moment où il est posé sur un mets, et le moment où il arrive devant le convive. Le remède était pourtant évident : il suffisait de plonger préalablement les cristaux dans de l'huile... et c'est ainsi que, pour Pierre Gagnaire, cette « invention » est l'une des plus intéressantes de toutes celles qui ont été proposées, au point qu'il y a des bols emplis d'huiles (colorées, parfumées) et différentes 
cristallisations de sel dans tous ses restaurants (Paris, Londres, Tokyo, Courchevel, Marrakech, Shanghai, Séoul, Montreux, Châteleillon, Dubaï, Nîmes...). En termes d'utilisation de la science, on en reste là toutefois à des amusements d'enfant. Mais ne nous étonnons pas que les critères d'appréciation des cuisiniers ne soient pas les mêmes que ceux des scientifiques: les critères culinaires sont techniques ou artistiques, alors que les critères des scientifiques sont... scientifiques.

Bien d'autres idées ont donc été mises en œuvre : le «chocolat chantilly ${ }^{7}$, qui est une mousse de chocolat, sans œuf, où l'on fait foisonner une émulsion de chocolat (Figure 3); la sauce Kientzheim ${ }^{8}$, qui est l'analogue d'une sauce mayonnaise, mais où l'huile est remplacée par du beurre noisette ; les gibbs (Figure 4), qui sont des soufflés faits en quelques dizaines de secondes ; ou encore les würtz, qui sont des mousses gélifiées (Figure 5)... À propos de ces deux dernières, on observera que le nom donné à l'invention est respectivement celui du physico-chimiste américain Josiah Willard Gibbs (1839-1903) et du chimiste alsacien Charles Adolphe Würtz (1817-1884). En effet, la plupart de mes inventions reçoivent le nom d'un chimiste que je veux honorer (Geoffroy, Lavoisier, Thénard, Braconnot, Gay-Lussac, etc.), car je souhaite faire connaitre au public les grands chimistes du passé, d'une part, et, d'autre part, lui faire penser que le crédit de mets délicieux revient à la chimie (ce qui n'est pas juste : il revient à celui ou celle qui a produit le mets).

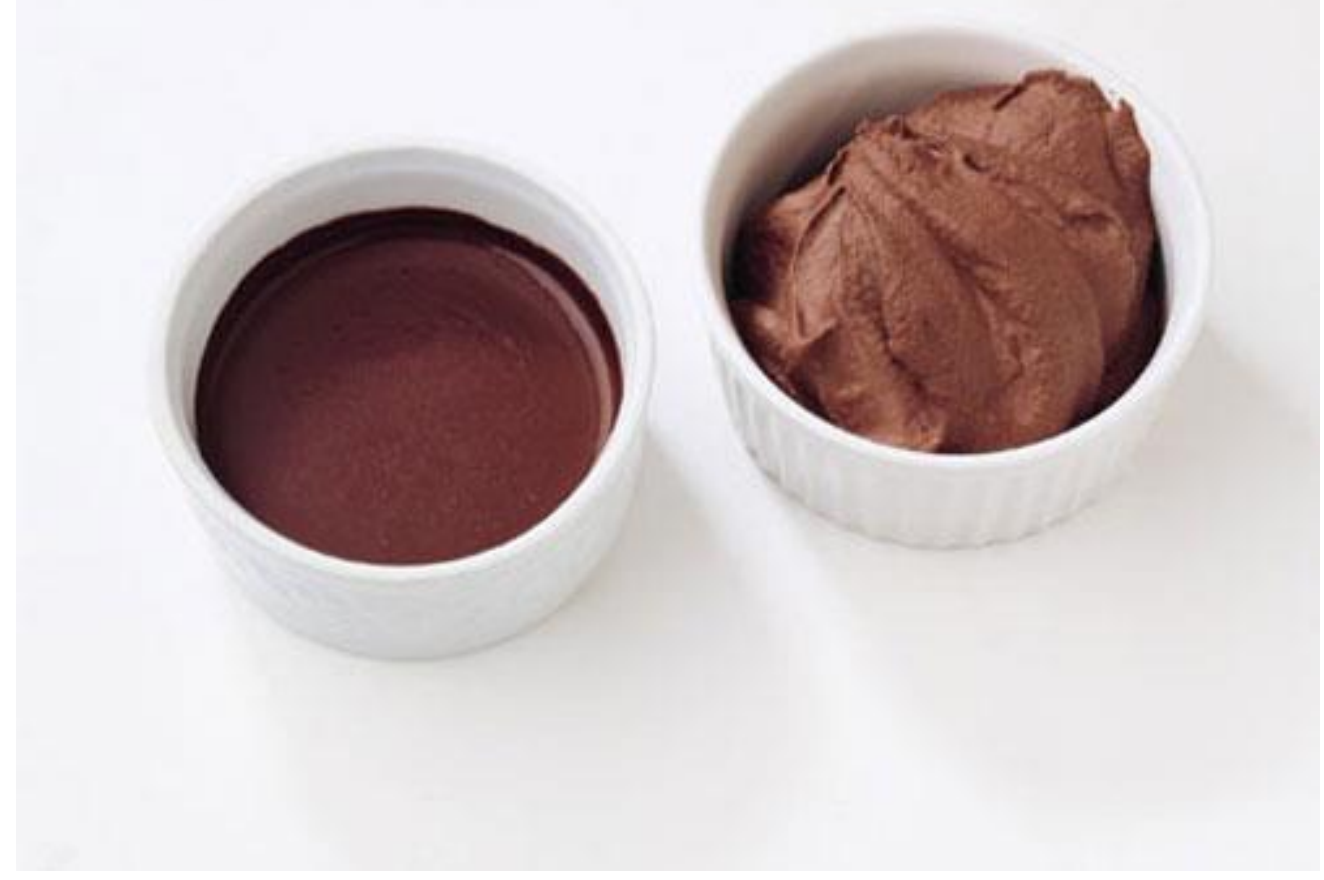

Figure 3. Un " chocolat chantilly " s'obtient à partir d'une émulsion de chocolat chaude que l'on fait foisonner, lors du refroidissement. De la même manière, on peut obtenir du foie gras chantilly, du beurre chantilly, du beurre noisette chantilly, du fromage chantilly... 


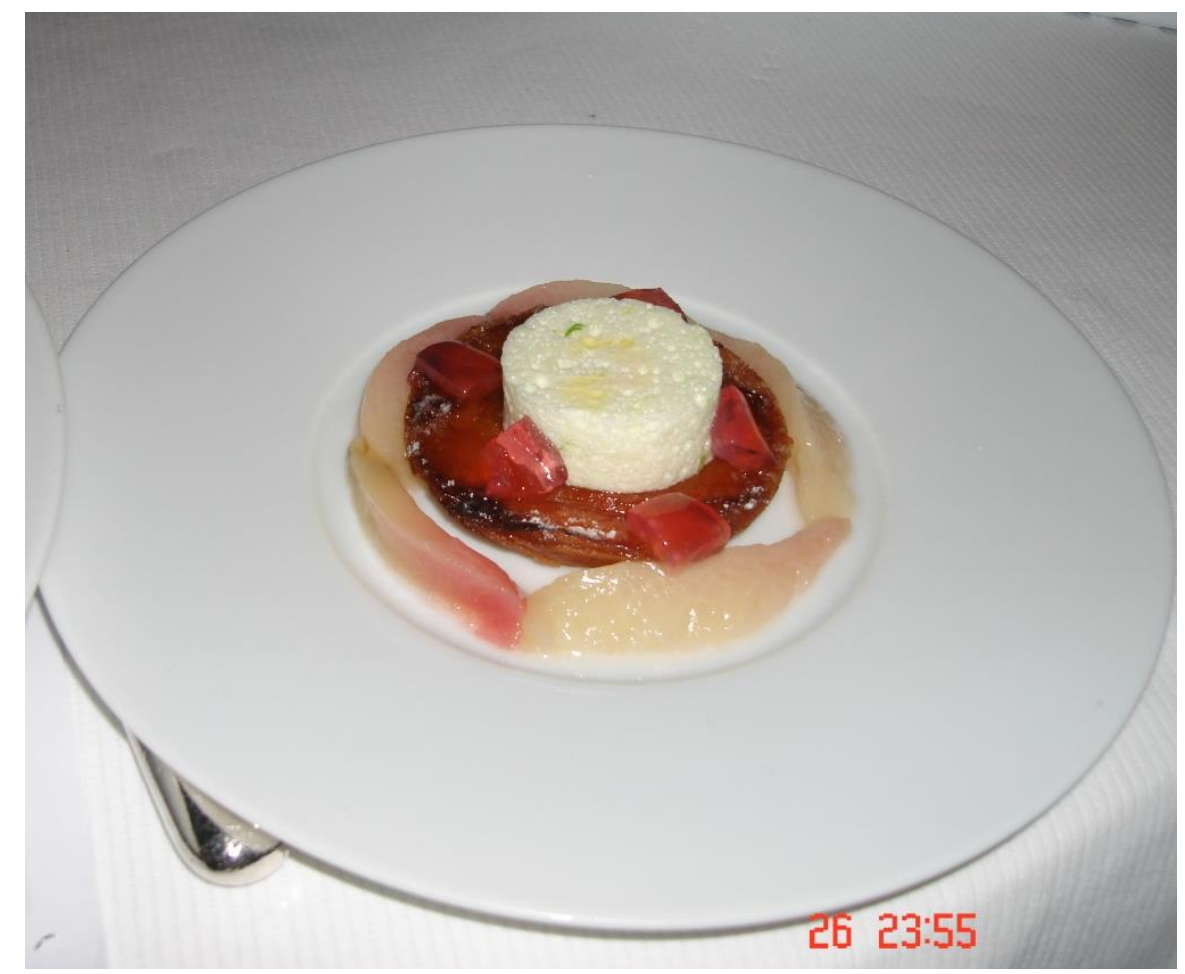

Figure 4. Un « gibbs » préparé par Pierre Gagnaire pour un repas de gala au restaurant Le Balzac, en 2002.

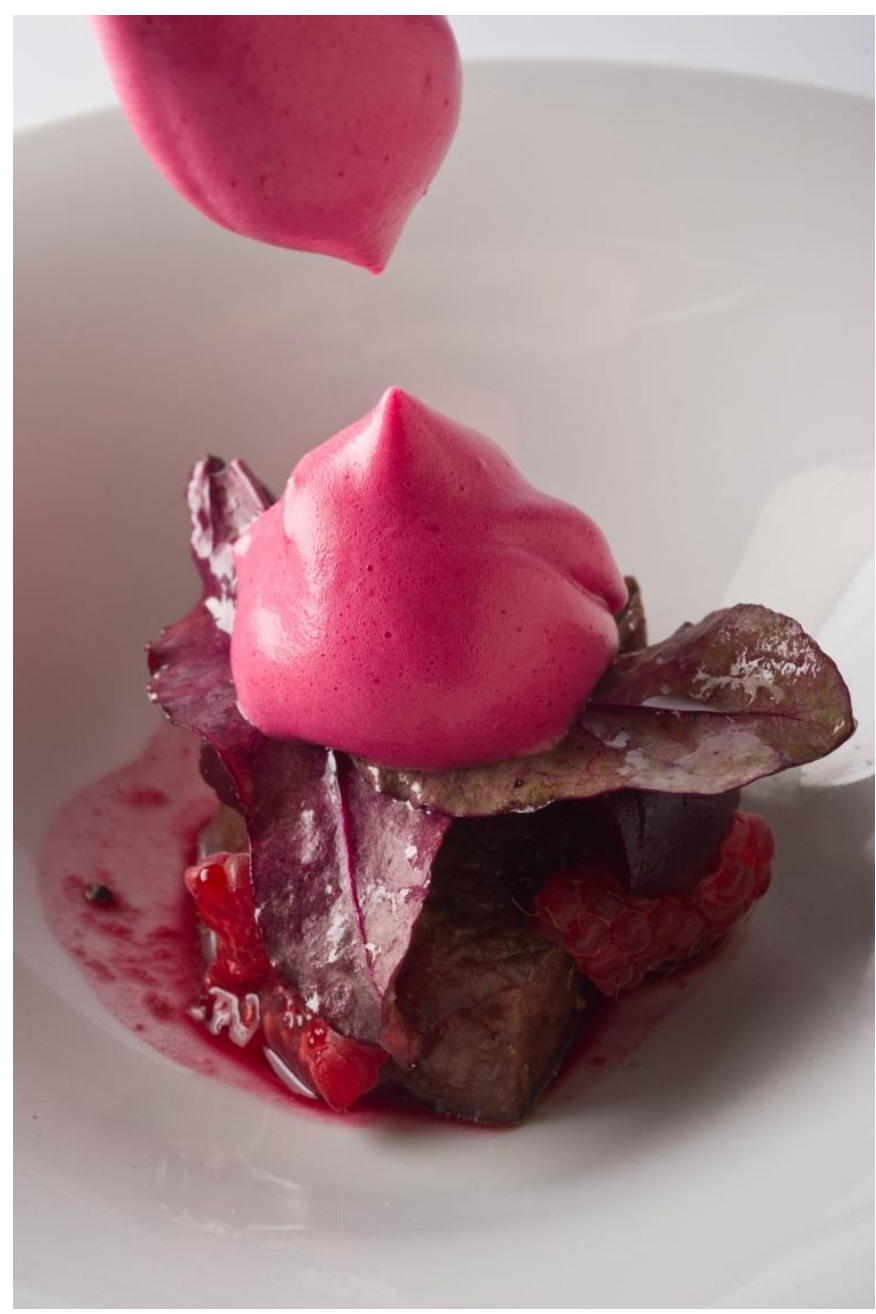

Figure 5. Un « würtz » : une mousse gélifiée. Ici, le würtz au cassis, réalisé par Pierre Gagnaire, est servi avec une pièce de bœuf. 


\section{De la « science », mais quelle science?}

À ce stade, prenons du recul et considérons les deux termes de «science et art», pour ce qui concerne l'activité culinaire. Pour l'art culinaire, nous l'avons évoqué, mais que penser d'une «science culinaire»? Je vais maintenant montrer pourquoi c'est soit une tautologie, soit une impossibilité.

Commençons par observer que le mot «science» désigne tout aussi bien un simple savoir technique qu'une science de la nature, ou une science de l'humain ou de la société. Comme le mot « art », d'ailleurs, qui n'a pas toujours eu l'acception qui le fait accoler à des Mozart ou à des Flaubert (on parlait ainsi des «arts chimiques » pour désigner l'application industrielle de la chimie $)^{9}$. Bref, le mot « science » a fait trébucher nombre de cuisiniers qui avaient une vision élevée de leur profession. Par exemple, Marie-Antoine Carême a écrit : «La cuisine se veut également une science ${ }^{10}$. Que cela signifie-t-il ? Si c'est la « science de la cuisine », au sens de savoir, comme on parle de «science du maître d'hôtel», de «science du cordonnier, alors on peut accepter l'idée proposée, même mal formulée, car les métiers techniques sont plein d'un immense savoir technique, et il n'y a pas lieu d'envisager une évolution particulière, de ce point de vue. Autrement dit, quand Carême dit que la cuisine est une science, c'est une évidence... maladroite, car la cuisine est d'abord une technique doublée d'une composante artistique. En revanche, si la « science » qu'il imagine est une science de la nature, alors il y a une impossibilité, puisque la cuisine est une technique (doublée d'une composante artistique), alors que les sciences de la nature sont des productions de connaissances.

Puis, quand Carême écrit : «La science culinaire est plus salubre à la santé des hommes que tous les doctes préceptes de ceux qui prolongent les maladies par spéculation », c'est bien, à nouveau, l'acception de savoir qu'il retient, pas celle de science de la nature. Quant à considérer si la cuisine est bien plus salubre que la médecine, c'est une prétention qui ne grandit pas son auteur.

Une génération après lui, les cuisiniers français Urbain Dubois, Émile Bernard, Jules Gouffé ou Joseph Favre ont poursuivi son idée, en évoquant l'utilisation de proportions précises, pour leurs réalisations, mais cela ne change pas la nature de leur activité, et leur rigueur ne transforme pas la production d'aliments en science de la nature pour autant. Favre, notamment, évoque une « cuisine scientifique », qui serait, «de toutes les sciences, celle qui s'attache à l'art de bien préparer les aliments ». La question demeure : cuisine scientifique avec scientifique au sens de savoir technique ? Ou de science de la nature ? À nouveau, cette citation est confuse. D'ailleurs, ce n'est pas le fait d'être précis qui fait qu'une activité est ou pas une science de la nature. Une cuisine précise est une activité technique précise, et rien de plus.

Auguste Escoffier s'est joint à ce concert un peu incohérent : « La cuisine, sans cesser d'être un art, deviendra scientifique et devra soumettre ses formules, empiriques trop souvent encore, à une méthode et à une précision qui ne laisseront rien au hasard. » Là encore, je propose de penser que cette proposition est soit fausse, soit tautologique. La cuisine ne deviendra jamais scientifique, au sens des sciences de la nature, parce que, je le répète, la cuisine est une production, et pas une recherche des mécanismes des phénomènes. Et Escoffier, à ma connaissance, n'a jamais manié de calcul, lequel, on le répète, est l'essence même des sciences de la nature ${ }^{11}$.

9 Cuvier G., 1989, Rapports à l'Empereur sur le progrès des sciences, des lettres et des arts depuis 1789. II. Chimie et sciences de la nature, présentation et notes sous la dir. d’Yves Laissus, préface par Denis Woronoff, Paris, Belin, (1810).

10 Carême M. A., 1847, L'art de la cuisine française au $19^{e}$ siècle, Chez l'Auteur, Paris. 
Plus près de nous, au $20^{\mathrm{e}}$ siècle, le microbiologiste Édouard de Pomiane introduisit le mot «gastrotechnie », mais on trouvera dans «Pourquoi la cuisine n'est pas une science ?» ${ }^{12}$ une analyse de la confusion intellectuelle qui conduisit à cette proposition. Pomiane fut célèbre de son temps... mais ses ouvrages ne sont en réalité que des livres de recettes par un amateur (éclairé) qui, quand il évoque des phénomènes physico-chimiques, écrit des absurdités. Par exemple, Pomiane propage l'idée selon laquelle on éviterait de pleurer si l'on mordait une cuiller en bois quand on épluche des oignons, mais l'expérience a réfuté cette idée. Il écrit avec beaucoup d'autorité qu'il faut un fouet en fil de fer, et un cul de poule en cuivre, pour faire foisonner des blancs d'œufs (produire des «blancs en neige »), parce que le rapprochement ferait un « effet pile », mais n'importe qui peut s'amuser à monter des blancs en neige avec un fouet en plastique dans un bol en plastique, système où il n'y aura aucun «effet pile ». Bref, le succès populaire de Pomiane, notamment associé à son émission de Radio Cuisine, n'est pas garant de la justesse de ses écrits, ni de ses réflexions sur la nature de son activité.

Souvent, aussi, le contexte dans lequel l'expression «science culinaire » est utilisée doit rapprocher cette dernière de celle - également fautive - de « science appliquée », si vigoureusement - et légitimement - combattue par Louis Pasteur : il y a des sciences, d'une part, et des applications des sciences, d'autre part, mais pas de « sciences appliquées » ${ }^{13} 1415$.

Et c'est ainsi que, pour éviter une faute de langage, nous avons nommé «gastronomie moléculaire et physique » la discipline scientifique (physico-chimie) qui explore les mécanismes des phénomènes qui s'observent lors de la préparation et de la consommation des mets. Là, la dénomination est parfaitement choisie, car le juriste Jean-Anthelme Brillat-Savarin, en 1825, a défini la gastronomie comme la connaissance raisonnée de tout ce qui se rapporte à l'être humain qui se nourrit ${ }^{16}$. Quand les études sont de chimie ou de physique, alors il s'agit bien de gastronomie moléculaire et physique, ce qui a été abrégé en gastronomie moléculaire.

Le nom de cette activité scientifique a été conjointement introduit par Nicholas Kurti (1908-1998) et moi-même, en 1988, alors que nous préparions les International Workshops on Molecular and Physical Gastronomy, et il fit le titre de ma thèse de sciences, soutenue en 1995 à la demande du président de l'Académie des sciences d'alors, Guy Ourisson. Le jury comprenait deux lauréats du prix Nobel, Jean-Marie Lehn et Pierre-Gilles de Gennes, mais aussi ce remarquable pharmacien et chimiste qu'était Pierre Potier, et d'autres collègues de renom : ces précisions, à nouveau, ne sont pas des manifestations de prétention, mais elles veulent indiquer que la communauté avait bien

12 This H., 2006, « Pourquoi la cuisine n'est pas une science ? », Sciences des aliments, 2006, 26 (3), 201-210).

13 « Souvenez-vous qu'il n'existe pas de sciences appliquées mais seulement des applications de la science ", Pourquoi le goût de la vendange diffère de celui du raisin, Comptes rendus du Congrès viticole et séricicole de Lyon, 9-14 septembre 1872, p. 4549 (séance du 11 septembre 1872), in Louis Pasteur, CEuvres complètes, tome 3, p. 464. Masson, Paris, 1924.

14 « Une idée essentiellement fausse a été mêlée aux discussions nombreuses soulevées par la création d’un enseignement secondaire professionnel ; c'est qu'il existe des sciences appliquées. Il n'y a pas de sciences appliquées. L'union même de ces mots est choquante. Mais il y a des applications de la science, ce qui est bien différent. Puis, à côté des applications de la science, il y a le métier, représenté par l'ouvrier plus ou moins habile. L'enseignement du métier a un nom dans toutes les langues. Dans la nôtre, il s'appelle l'apprentissage, que rien au monde ne peut remplacer ", in Louis Pasteur, CEuvres complètes, Tome 7, p. 187 : Note sur l'enseignement professionnel, adressée à Victor Duruy, 10 nov. 1863.

15 « Non, mille fois non, il n'existe pas une catégorie de sciences auxquelles on puisse donner le nom de sciences appliquées. Il y a la science et les applications de la science, liées entre elles comme le fruit à l'arbre qui l'a porté. », p. 215, article « Pourquoi la France n'a pas trouvé d’homme supérieur au moment du péril », paru dans Le Salut public, Lyon, mars 1871, et dans La Revue Scientifique, 22 juillet 1872, $2^{\mathrm{e}}$ série, in Louis Pasteur, CEuvres complètes tome 7. 
compris que la gastronomie moléculaire était une activité scientifique, et non pas de la cuisine. À ce jour, après quelques décennies de travail, il y a 34 groupes de recherche en gastronomie moléculaire et physique dans le monde, et il s'en crée régulièrement de nouveaux. C'est sur la base de cette communauté internationale que nous avons récemment publié un énorme Handbook of Molecular Gastronomy (894 pages 150 chapitres, 150 auteurs de 23 pays) ${ }^{17}$. Et notre communauté se retrouve tous les ans pour des workshops, des séminaires, etc. L'activité scientifique est intense, et nous parlons bien ici de science de la nature.

Pour les sciences de la nature, pour la gastronomie moléculaire en particulier, l'objectif est donc d'explorer les mécanismes des phénomènes avec la méthode des sciences de la nature, qui passe par (1) identification d'un phénomène, (2) caractérisation quantitative du phénomène, (3) réunion des données de mesure en modèles, équations, formules (ce que l'on nommait naguère des lois), (4) constitution de théorie par induction de concepts nouveaux, puis (5) recherche de conséquences théoriques testables expérimentalement, et (6) tests expérimentaux de ces prévisions théoriques, en vue de trouver des réfutations à la théorie, qu'il faudra ensuite améliorer.

Rien d'original, donc: nous avons une activité scientifique parfaitement habituelle, et, peut-être même, encore plus «raide» que nécessaire, car nous avions initialement la crainte d'être soupçonnés de faire une espèce de science au rabais, une technologie... mais seulement par des collègues médiocres, car les autres, les Jean-Marie Lehn ou les Pierre-Gilles de Gennes avaient bien montré publiquement qu'ils n'avaient pas ce préjugé.

Pour la gastronomie moléculaire, après des hésitations à propos du programme scientifique de la discipline, se pose une question de stratégie scientifique (commune à toutes les sciences de la nature) : comment choisir, parmi les phénomènes bien établis, ceux qui pourront conduire à des découvertes scientifiques? La question est discutée dans This, $2017^{18}$. La première étape de l'activité scientifique (l'identification des phénomènes) a été et est restée essentielle, car nombre d'idées transmises oralement ou par écrit, dans le milieu culinaire (professionnel ou non) sont fausses. Après des travaux initiaux souvent consacrés à tester les dictons, on-dit, trucs, astuces, tours de main, proverbes (tout ce que nous avons proposé de nommer des "précisions culinaires »), les séminaires mensuels de gastronomie moléculaire organisent des tests expérimentaux, en présence des professionnels, ce qui conduit à nombre de réfutations d'idées classiques, en même temps que l'on arrive parfois à des phénomènes insoupçonnés ${ }^{19}$.

Ici, l'intérêt scientifique et l'intérêt technique se rejoignent, mais, ensuite, il y a de moins en moins de points communs, et seules les applications de la gastronomie moléculaire peuvent intéresser le monde culinaire, et principalement pour la partie technique, et certainement pas pour la partie artistique. D'ailleurs, quand on discute les applications des sciences de la nature, il est bon de se souvenir que ces applications sont au moins de deux sortes : il y a des applications techniques, et aussi des contributions à l'image que les humains ont du monde (par l'enseignement), notamment en examinant précisément les dénominations des objets culinaires, selon le bon principe, dû à Condillac et Lavoisier, selon lequel les mots et la pensée vont de pair ${ }^{20}$. Et c'est ainsi que la gastronomie moléculaire a permis de réviser nombre d'idées fausses, de confusions entre des systèmes mal

17 Burke R, Kelly A., Lavelle C., This Vo Kientza H. (eds), 2021, Handbook of Molecular Gastronomy, CRC Press, Boca Raton, Florida.

18 This H., 2017, "Molecular gastronomy, as a prototype for discussing the issue of scientific strategy", Irish Chemical News, 5, December 2017, 56-71.

19 http://www2.agroparistech.fr/-Les-Seminaires-de-gastronomie-moleculaire-

20 Lavoisier A. L., 1791, Traité élémentaire de chimie, Cuchet, Paris. 
nommés : des mousses confondues avec des émulsions, des émulsions confondues avec des gels, etc.

Notamment la compréhension physico-chimique des systèmes rencontrés en cuisine classique conduit à des rénovations techniques, mais aussi à des innovations. Par exemple, le formalisme des systèmes dispersés, introduit en 1991, a permis une classification des 451 sauces classiques françaises, ce qui a non seulement introduit de la cohérence dans un système très désordonné, mais, de surcroît, a conduit à des innovations, en faisant apparaître des systèmes qui ne figuraient pas dans la liste classique (par exemple, des « veloutés foisonnés ») ${ }^{21}$ : c'est une preuve que l'empirisme, qui a toujours prévalu pour les techniques et l'art culinaires n'a pas suffi, malgré les siècles, et peut-être en partie en raison de la néophobie alimentaire qui caractérise l'être humain, à explorer systématiquement le monde des possibilités techniques.

\section{Une indispensable rénovation technique}

Bien différente de la gastronomie moléculaire est cette activité, que j'ai nommée «cuisine moléculaire » : comme le premier terme du nom l'indique, c'est bien une activité culinaire, de préparation des aliments. En réalité, c'est dès le début des années 1980 que Nicholas Kurti, moimême et quelques autres (Peter Barham, à Bristol ; Jean Matricon, à Paris ; Hugo et Béatrice Palma à Palerme) avons cherché (à côté de notre activité scientifique de gastronomie moléculaire) à rénover les techniques culinaires, en faisant venir en cuisine des ustensiles présents dans les laboratoires de chimie, de physique ou de biologie. Et c'est cela qui a été nommé "cuisine moléculaire », bien plus tard (en 1999), pour éviter que les cuisiniers qui cuisinaient ainsi de façon moderne (Raymond Blanc à Oxford, Ferran Adria à Rosas, Heston Blumenthal à Londres, etc.) ne soient vexés (surtout devant la presse) qu'on leur dise que non, ils ne faisaient pas de gastronomie moléculaire ; l'emploi de l'expression «cuisine moléculaire », certes grammaticalement et sémantiquement fautive, fut un compromis introduit dans l'urgence d'un tournage de la télévision.

Effectivement il y a eu -et il demeure- beaucoup de confusions entre gastronomie moléculaire et cuisine moléculaire, notamment parce que Nicholas Kurti et moi-même invitions des cuisiniers à nos workshops internationaux, à Erice (Sicile) : nous voulions avoir avec nous des professionnels de la cuisine pour mieux appréhender les phénomènes que nous souhaitions explorer. Cela, plus la confusion assez générale entre cuisine d'apparat ou artistique, et gastronomie avait suffi à créer une confusion qui subsiste souvent aujourd'hui.

Pourtant les choses sont claires : la gastronomie moléculaire est une activité scientifique, au sens des sciences de la nature, tandis que la cuisine moléculaire est une activité culinaire, technique ou artistique, fondée sur l'emploi d'ustensiles venus des laboratoires. Et il y avait lieu de rénover les techniques culinaires ! Quand on pense que nous utilisons encore des casseroles, des fouets... Pourquoi n'a-t-on pas mis l'objectif en premier, avant de mettre l'outil ? Il faut avouer que si des d'Arcet, des Lavoisier, des Geoffroy, au $18^{\mathrm{e}}$ siècle ont exploré quelques préparations culinaires, si des Braconnot, des Payen et quelques autres ont poursuivi le travail au siècle suivant, rien de tout leur apport n'était arrivé en cuisine. D’ailleurs on peut observer que les préparations de la « nouvelle cuisine », dans les années 1970, étaient déjà dans des livres de cuisine des siècles passés, et que le Guide culinaire, publié pour la première fois avant le début du $20^{\mathrm{e}}$ siècle $^{22}$, restait la référence de l'enseignement national culinaire. Alors qu'un manuel de chimie de plus de 10 ans est périmé !

21 This H., 2009, "Molecular Gastronomy, a chemical look to cooking” , Accounts of Chemical Research, May 2009, vol. 42, N5, p. 575-583. 
Pourquoi une telle pérennité des pratiques culinaires? Pourquoi, dans les cuisines, a-t-on continué à transmettre des idées techniques fausses aux apprenants? Dans les années 1990, une grande école hôtelière suisse enseignait encore que les blancs en neige étaient mieux foisonnés quand les œufs étaient battus toujours dans le même sens, et l'on a même vu une inspectrice de l'Éducation nationale déclarer publiquement qu'elle ratait les mayonnaises lors de ses menstruations, alors que le foisonnement n'est pas sensible au sens de battage, dans le premier cas, et que les menstruations n'ont évidemment pas les effets «magiques» évoqués. Surtout, fut enseignée pendant plus d'un siècle une théorie fausse des cuissons, où il y aurait eu des « concentrations » (pour le rôtissage, par exemple) et des «expansions » (pour les bouillis); l'idée ne fut éradiquée qu'en 2004 par la $17^{\mathrm{e}}$ Commission paritaire des programmes, lors de la rénovation des certificats d'aptitude professionnelle (CAP) hôtellerie-restauration.

Malgré les progrès apportés par la cuisine moléculaire, la rénovation technique n'est pas terminée. Nous avons introduit des thermocirculateurs pour chauffer à des températures précises, des siphons pour produire des mousses, des sondes à ultrasons pour réaliser des émulsions, etc., mais de nombreuses opérations restent effectuées de façon traditionnelle, et sont donc susceptibles d'être rationalisées et perfectionnées. Pensons que la clarification d'un bouillon de viande impose plusieurs heures de travail, entre le stockage en chambre froide pour obtenir une solidification des graisses que l'on retire, puis la remise à l'ébullition avec addition de blanc d'œuf battu en neige, afin de piéger les impuretés lors de la coagulation des protéines («clarification»)! Une ampoule à décanter et un filtre bien calibré de laboratoire de chimie, associé à un système de vide, conduisent en quelques minutes seulement à des bouillons plus limpides. Et l'on observe ici que ce ne sont pas les sciences de la nature stricto sensu qui sont utiles, mais seulement des réflexions technologiques bien conduites.

On terminera cette discussion de la «cuisine moléculaire » en observant que la langue anglaise permet une subtilité que le français ne permet pas : elle distingue molecular cooking et molecular cuisine. Si la première désigne une technique, la seconde nomme un style culinaire qui est né de l'utilisation de la nouvelle technique. Quand les cuisiniers ont disposé de siphons, par exemple, ils ont produit des mousses d'une consistance très différente de celles qu'ils obtenaient avec les fouets (Figure 6). Quand on a utilisé des thermocirculateurs, on a eu des consistances de viande, de poisson ou d'œufs très différentes de celles que l'on obtenait classiquement avec des casseroles. Quand on a utilisé de l'azote liquide, on a non seulement pu congeler des préparations nouvelles (alcools, huiles dont on a fait des poudres, etc.), mais, aussi, produire des systèmes impossibles à réaliser par le passé, telles des sphères contenant des liquides qui contenaient des sphères qui contenaient des liquides (Figure 7). Là encore, aucun apport des sciences de la nature, mais seulement de la technologie, c'est-à-dire l'amélioration de techniques ${ }^{23}$. 


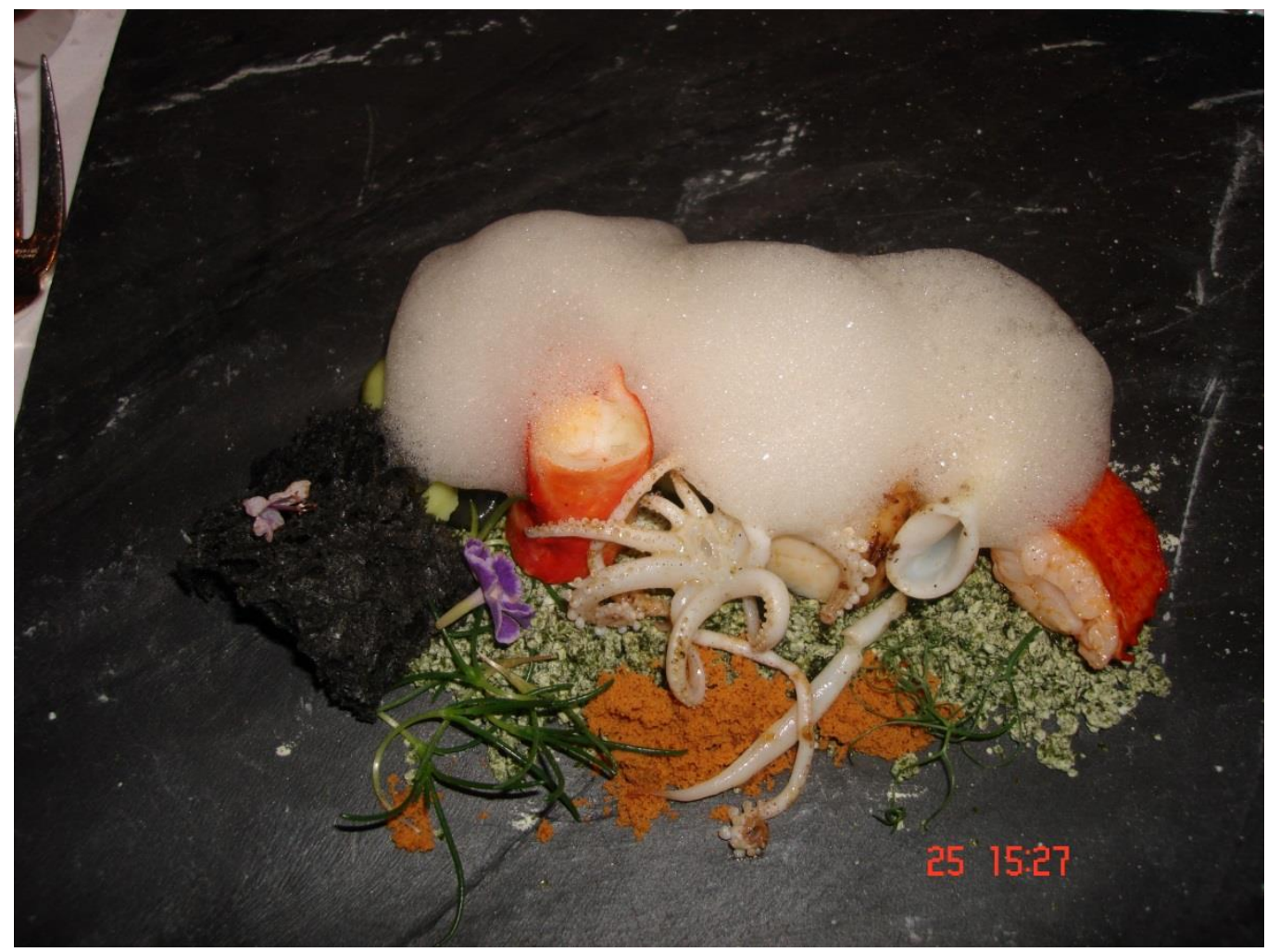

Figure 6. Sur ce plat intitulé "Under the sea », du chef Uwe Opocensky (Hong Kong), on voit une mousse obtenue au siphon, ainsi que de l'huile d'olive en poudre, produite à l'aide d'azote liquide.

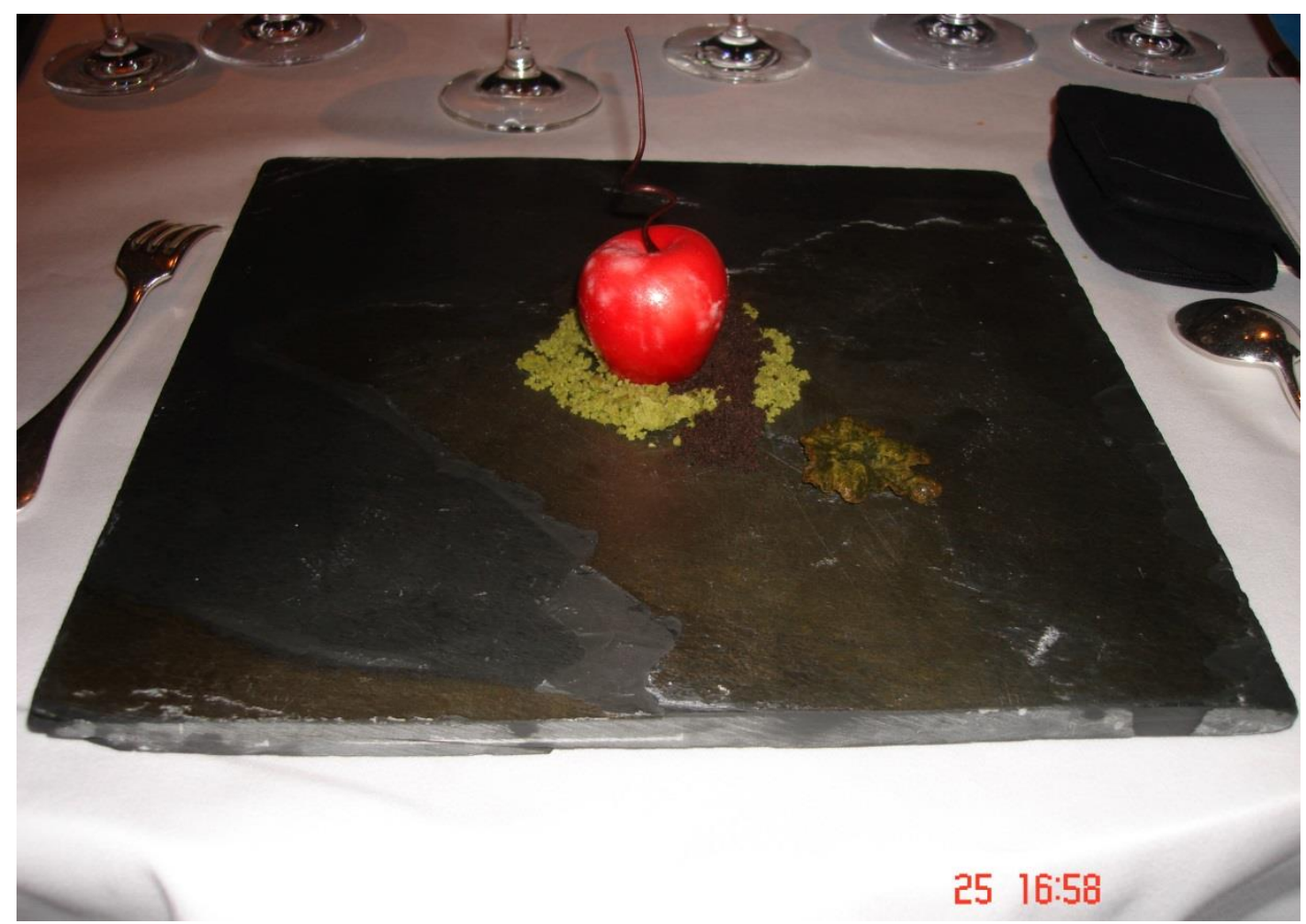

Figure 7. Cette " pomme » servie par le chef Uwe Opocensky, dans son restaurant du Mandarin Oriental, à Hong Kong, est un emboîtement de liquides et de solides, que l'on obtient à l'aide d'azote liquide : il suffit de plonger alternativement une baguette (ici, une gousse de vanille) dans de l'azote liquide et dans une solution aqueuse (jus de fruit, par exemple), jusqu'à obtenir une boule glacée que l'on trempe dans du chocolat fondu ; l'ensemble est alors, à nouveau, alternativement trempé dans l'azote liquide et dans une solution aqueuse, avant d'être trempé dans du chocolat fondu. Lors du réchauffement, la solution aqueuse congelée redevient liquide. 


\section{L'analyse esthétique, pour de nouveaux courants d'art culinaire}

Après la cuisine moléculaire, d'autres analyses ont conduit à d'autres propositions artistiques : mon livre intitulé La cuisine, c'est de l'amour, de l'art, de la technique ${ }^{24}$ est, ainsi, un traité d'« esthétique culinaire », le terme « esthétique » désignant justement la branche de la philosophie qui explore le « beau ». Et c'est ainsi qu'en transposant les courants des autres arts à la cuisine, a été proposée toute une série d'idées nouvelles que nous ne pourrons pas décrire ici.

Évoquons seulement la « cuisine abstraite », qui a résulté de l'analyse critique d'une idée fausse, longtemps propagée par les gastronomes, selon laquelle les mets seraient bons «quand ils ont le goût de ce qu'ils sont». On commencera par observer que l'on doit éliminer des discussions rationnelles relatives à l'alimentation, des termes tels que «bon » ou « meilleur », qui n'ont aucune validité réelle : n'est bon que ce que l'on aime manger hic et nunc; et, pour le «meilleur», on n'oubliera pas que l'intransitivité des choix doit empêcher toute utilisation du terme. D'autre part, on rappellera utilement que la règle proposée n'a pas de validité artistique ${ }^{25}$, sans compter que l'oukase classique était manifestement faux : non seulement le «poulet rôti » n'a pas le goût du poulet (cru), mais, d'autre part, de même que deux peintres différents produiront deux «Vierges à l'enfant » différentes, deux artistes culinaires produiront deux versions bien distinctes du «poulet rôti », avec deux goûts différents, qui ne pourront donc pas être le goût du poulet rôti. C'est d'ailleurs l'occasion d'observer que, pour des raisons qu'il est important d'analyser ${ }^{26}$, l'art culinaire était considérablement en retard par rapport aux autres arts, sans doute en raison de la néophobie alimentaire, laquelle est une prudence, face à une « nature » qui n'a pas les vertus que lui prêtent les plus naïfs d'entre nous.

Le champ artistique étant ouvert, l'ouvrage cité plus haut a proposé de transposer en cuisine les courants artistiques des autres arts, et c'est ainsi que fut imaginée une «cuisine abstraite », qui ne « représente plus » : il s'agit de faire « bon » sans que l'on sente le goût des ingrédients, tout comme des peintures de Paul Klee ou de Kandinsky font usage de points et lignes, au lieu de représenter des montagnes, des personnages, etc. ${ }^{27}$. À ma connaissance, Pierre Gagnaire est le seul à avoir produit de tels plats abstraits, ce qui nécessite une virtuosité technique alliée à une intuition artistique (Figure 8).

24 This H., Gagnaire P., 2010, La cuisine, c'est de l'amour, de l'art, de la technique, Odile Jacob, Paris.

25 Gauquelin A., op. cit.

26 This H., 2019, Mon histoire de cuisine, Belin, Paris.

27 Kandinsky V., 1991, Point et ligne sur plan, Gallimard (Folio), Paris. 


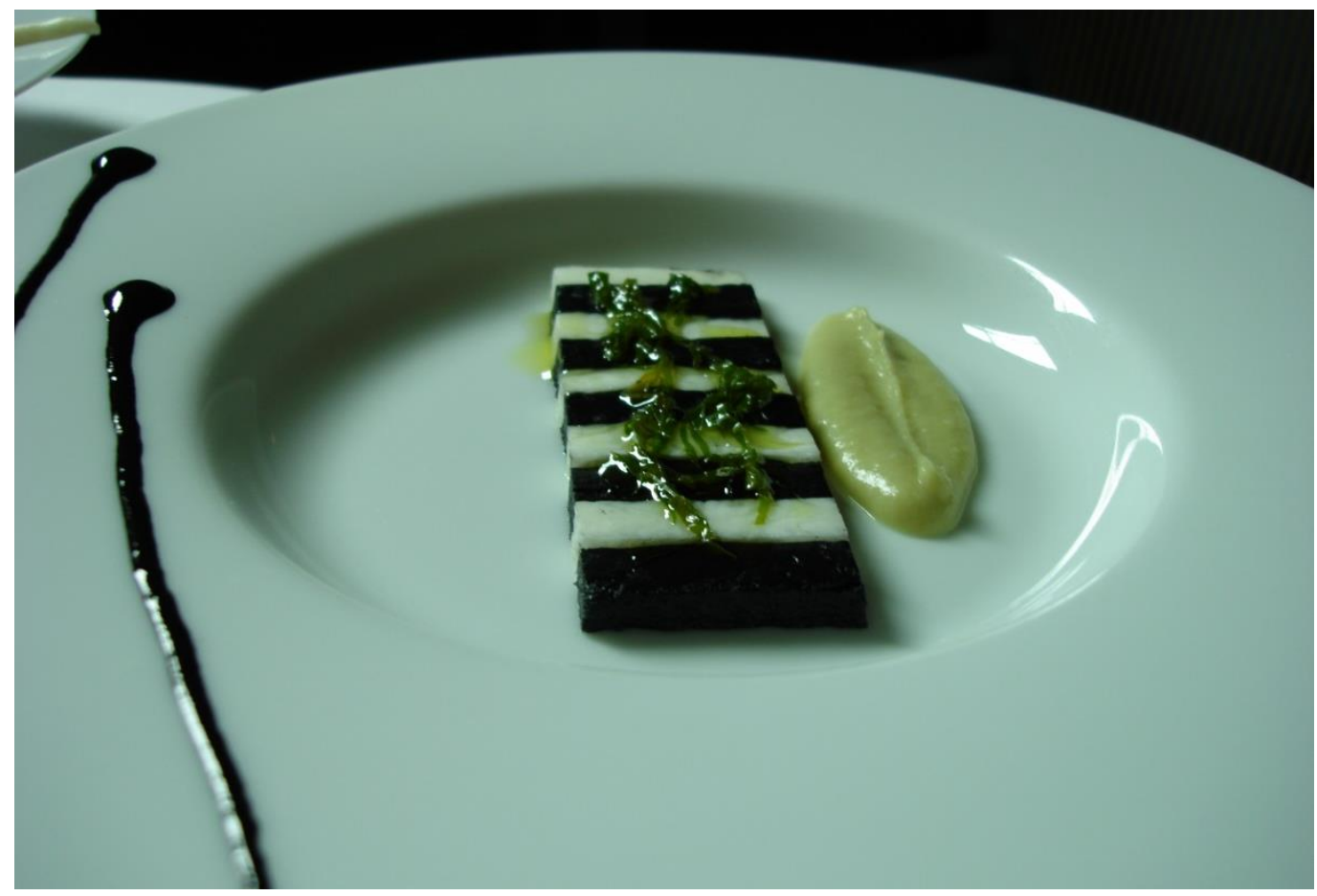

Figure 8. Un plat de cuisine abstraite servi par Pierre Gagnaire à Paris, en 2015.

\section{La cuisine de synthèse, nommée « cuisine note à note »}

La dernière grande idée en date est la «cuisine de synthèse », que j'ai proposée en 1994 et que j'ai surnommée «cuisine note à note », en vue de faciliter son acceptation par un public qui craint « la chimie », confondant la science de la nature qui est ainsi désignée et des composés qui sont fabriqués à partir des connaissances données par cette science ${ }^{28}$. On reconnaît dans la dénomination de « cuisine note à note » une analogie avec la musique, et c'est volontaire, car il fallait éviter des confusions entre les arts et les sciences.

Pour bien situer cette cuisine de synthèse, il est bon de filer la comparaison historique entre la musique et la cuisine. Il y a deux siècles, environ, on faisait de la musique avec des violons ou des flûtes... tandis que l'on cuisinait avec des légumes, des viandes, des poissons, des œufs, des fruits. De même que l'utilisation d'une flûte donne d'emblée un son de flûte, l'utilisation d'une carotte donne d'emblée un goût de carotte. Puis, il y a environ un siècle, les travaux remarquables du mathématicien Joseph Fourier et d'autres conduisirent à comprendre que les sons peuvent être considérés comme des sommes d'ondes sonores de fréquence pure ; à la même époque, les chimistes commencèrent l'analyse des ingrédients alimentaires, comprenant bientôt que ces derniers étaient des assemblages de composés tels qu'eau, protéines, lipides, saccharides, etc. Il y a 50 ans, apparurent les premières musiques de synthèse, champ qui a tant progressé que l'on trouve aujourd'hui des synthétiseurs dans des magasins de jouets, pour une dizaine d'euros, et que les musiques modernes font un large usage de ces synthèses. N'était-il pas évident qu'il fallait chercher le pendant culinaire de cette musique de synthèse ? C'est ce que j'ai proposé en 1994 : produire des mets en utilisant des composés purs, au lieu d'utiliser les ingrédients classiques.

On se rendra compte de l'audace de la proposition en considérant que Nicholas Kurti, qui fit une conférence révolutionnaire en 1969, à la Royal Institution of London, écrivait: "We shall see examples of both these precepts later, but let me first explain why I should speak about the physicist in the kitchen, and not about the chemist in the kitchen, or generally, about the scientist in the kitchen. My main reason for limiting my subject to physics is that the contributions of the chemists 
to culinary art and so many other activities designed to increase our sensual pleasures are well known. The chemists analyse and explain fragrancies and flavours and other invent new ones: a vast chemical industry is concerned with producing dyes to please our eyes." ${ }^{29}$ Il n'imaginait pas que l'on puisse non pas seulement limiter les apports de la « chimie » (il confond la science avec la production technique) à des composés odorants ou colorés, et c'est un fait que ma proposition de la cuisine de synthèse lui paraissait si exorbitante qu'il cosigna avec hésitation le texte où j'avais évoqué cette possibilité.

Quoi qu'il en soit, la cuisine note à note se développe aujourd'hui dans des restaurants de nombreux pays (Figure 9), et l'on assiste au même lent démarrage que pour la cuisine moléculaire dans les années 1980. Des actions de formation s'imposent, ainsi que la production des composés utilisables pour cette nouvelle technique, la mise au point de « recettes », qui évitent au praticien des réflexions qu'il n'est pas toujours capable de faire. Par exemple, le monde culinaire n'a pas souvent de connaissances sur les données toxicologiques des composés, et, d'autre part, il ne sait pas souvent faire les dilutions nécessaires pour utiliser des composés odorants purs dont la concentration d'usage doit être de quelques parties par million ou par milliard.

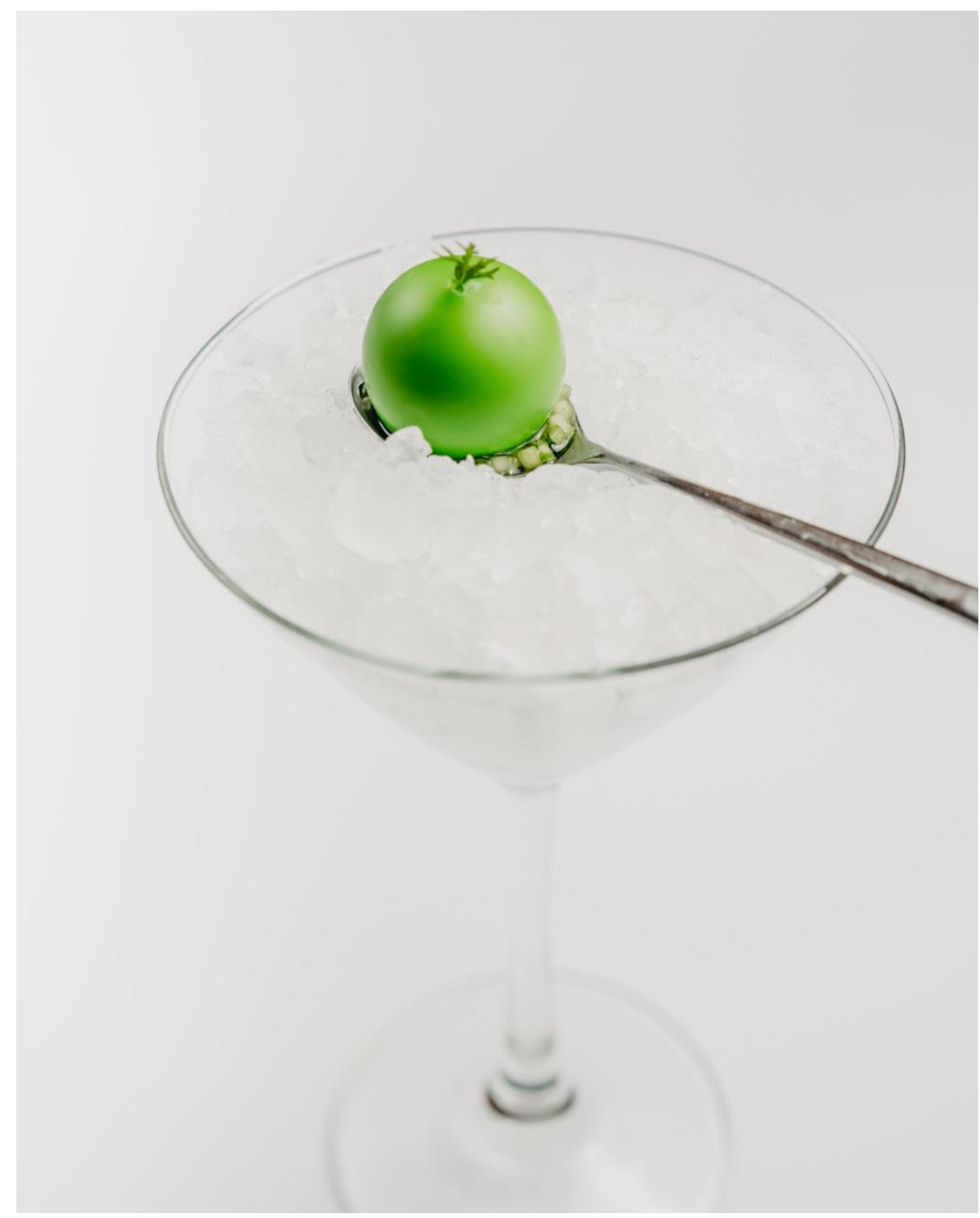

Figure 9. Un plat de cuisine note à note servi par le chef franco-italien Andrea Cammastra, dans son restaurant Senses, à Varsovie (Pologne). 


\section{La passerelle de la technologie}

Dans l'initiative présentée en début d'article comme dans les autres propositions, le scientifique qui participe aux transferts est en position de technologue, et non de scientifique, tandis que le cuisinier est d'abord en position de technicien, avant de se livrer, plus tard, à son travail artistique. Il y a donc nécessairement ce pont technologique, que l'on peut aujourd'hui mieux analyser qu'on ne l'a fait par le passé. On peut notamment distinguer une technologie «locale », celle du technicien qui a une réflexion technologique sur son travail, et une technologie «déportée », quand elle fait usage d'autres connaissances et compétences que celles du technicien. Cela est analysé dans le livre Cours de gastronomie moléculaire numéro 1 : sciences, technologie, techniques, quelles relations? (This, 2011).

Mais si l'on oublie cette passerelle, la science peut-elle bénéficier de l'art, et l'art de la science, pour le champ culinaire ? Pour le premier cas, que l'on me pardonne de ne guère voir d'exemples, alors que l'inverse n'est pas vrai : par exemple, quand j'avais indiqué à mon ami Pierre Gagnaire comment faire venir du goût dans les solides, en utilisant le phénomène de capillarité (conduisant à des préparations que j'avais nommées des «shitao », en l'honneur du peintre chinois éponyme), Pierre eut l'idée d'utiliser des navets ainsi préparés pour peindre l'assiette (Figure 10), ce qui était une nouvelle démonstration que l'art dépasse la technique !

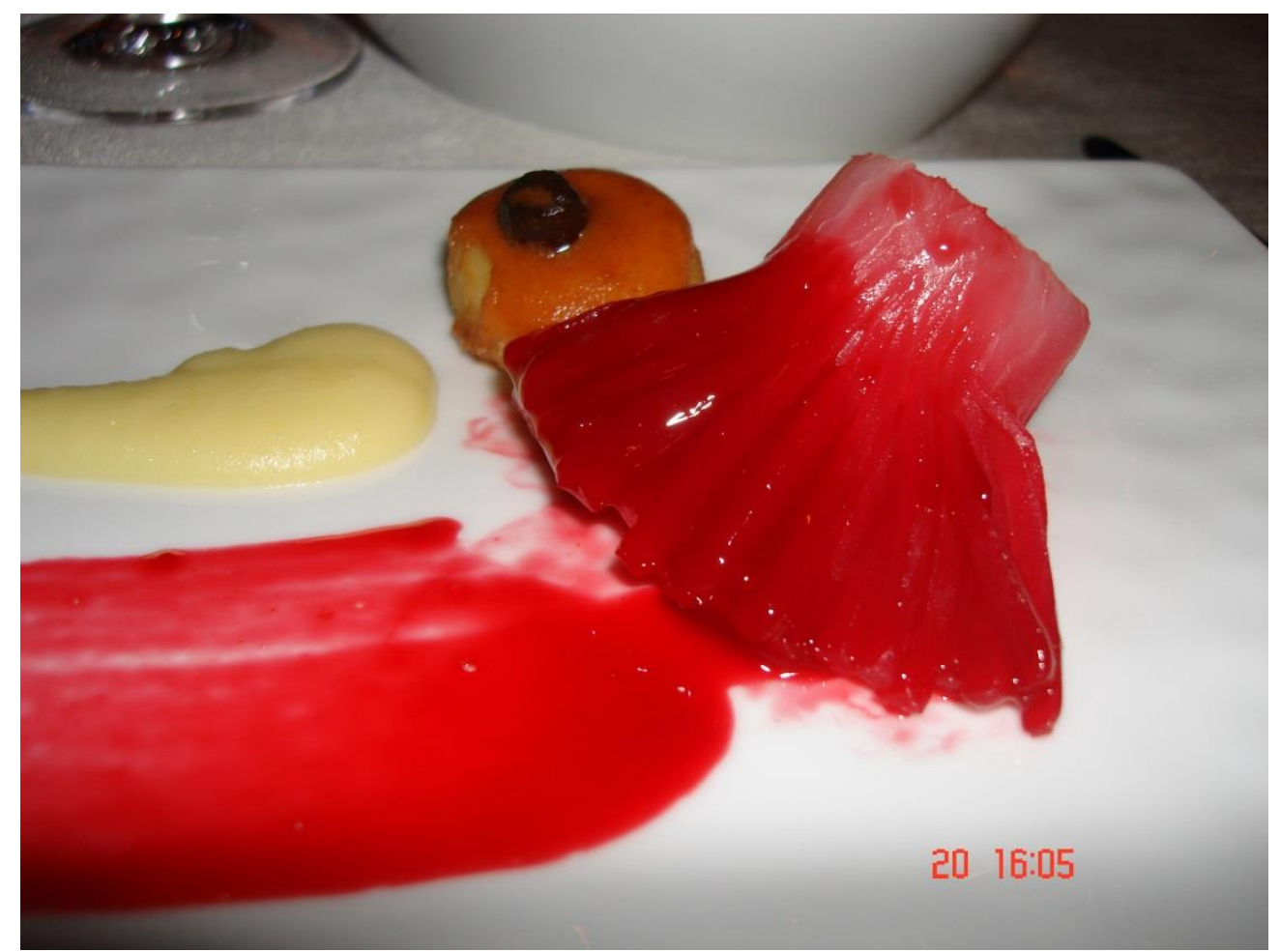

Figure 10. Un shitao : une proposition technique de $H$. This mise en art culinaire par Pierre Gagnaire pour le repas où fut servi le premier plat de cuisine note à note, à Hong Kong, en 2009.

Finalement, il pourra être intéressant d'observer que les enseignements du Bauhaus ont largement été mis à profit, non seulement pour des idées ponctuelles comme la proposition de la cuisine abstraite, mais, aussi, pour l'idée bien plus fondamentale d'exposer les jeunes artistes à des formations qui incluent des connaissances scientifiques.

Car on part de loin : je me souviens de cette émission de télévision, en 1992, où j'avais montré un sorbet à l'azote liquide (de la cuisine moléculaire, donc ; et non pas de la gastronomie moléculaire), et qui avait choqué des professionnels, moins pour l'emploi de l'azote liquide que par le mélange de basilic et de citron vert qui avait été glacé ; les professionnels observaient que l'association n'était pas classique, et ils s'interrogeaient sur sa toxicité éventuelle, partant de l'idée (fausse) selon 
laquelle la tradition aurait sélectionné des préparations sûres... et oubliant que nombre d'ingrédients classiques (basilic, estragon, noix muscade, etc.) ont des toxicités qui devraient faire reconsidérer leur emploi. À l'époque, les enseignements culinaires étaient si dépourvus de cours de science, pour les élèves comme pour les professeurs, que la crainte que la chimie (la science confondue avec ses applications) ne nous empoisonne était omniprésente. Avec l'aide de l'inspection générale hôtellerie restauration, nous avons introduit des programmes de formation des professeurs, nous avons proposé l'organisation d'ateliers expérimentaux avec les élèves, en vue de former des cuisiniers plus éclairés que par le passé.

Et quand il m'est demandé s'il faut être chimiste (gastronome moléculaire, notamment) pour être cuisinier, je réponds évidemment que non, puisqu'un chimiste explore les mécanismes des phénomènes par la méthode des sciences de la nature, alors qu'un cuisinier, s'il a évidemment le droit de s'intéresser aux sciences de la nature, a pour objectif de produire des œuvres d'art, culinaire en l'occurrence. 